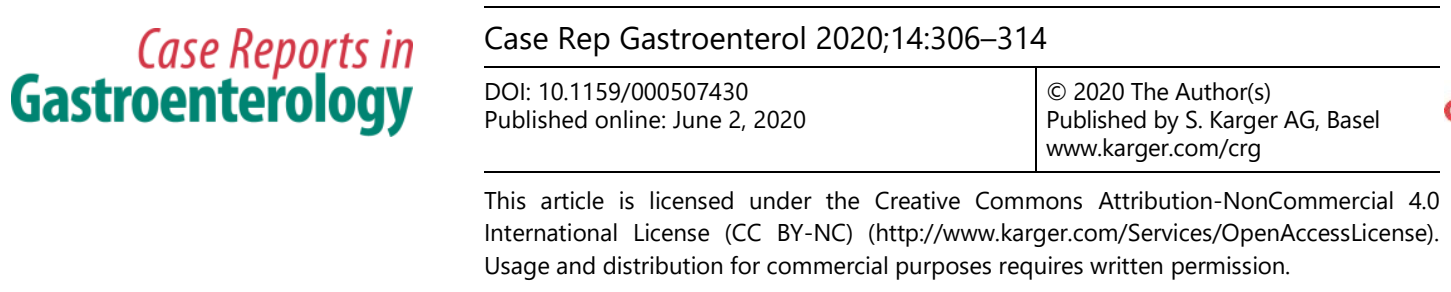

\title{
Groove Pancreatitis Associated with Transient Liver Injury Mimicking Ampullary Neoplasm
}

\author{
Elrazi Awadelkarim Hamid Ali ${ }^{a} \quad$ Ahmed Emad Mahfouz ${ }^{b}$ \\ Akhnuwkh Jones ${ }^{a}$ Abdelatif Abdelmola ${ }^{c}$ Mohamed A. Yassin ${ }^{d}$ \\ aDepartment of Internal Medicine, Hamad Medical Corporation, Doha, Qatar; \\ bepartment of Radiology, Hamad Medical Corporation, Doha, Qatar; 'Department of \\ Gastroenterology, Hamad Medical Corporation, Doha, Qatar; ${ }^{d}$ Department of Hematology \\ and Oncology, Hamad Medical Corporation, Doha, Qatar
}

\section{Keywords}

Jaundice $\cdot$ Ampullary cancer $\cdot$ Para-duodenal pancreatitis · Liver dysfunction

\begin{abstract}
Groove pancreatitis is an unusual form of pancreatitis characterized by fibrous inflammation and pseudo-tumor in the area around the head of the pancreas. The underlying etiology is unknown but is strongly linked to alcohol abuse. We report a 52-year-old male smoker with hypertension, asthma, and alcohol abuse who was admitted with severe epigastric pain radiating to the back. He was found to have acute pancreatitis. A computed tomography scan of the abdomen showed a mass lesion in the peri-ampullary region. MRI of the abdomen revealed dilated common bile duct and duodenal mass and features suggestive of groove pancreatitis. During the hospital stay, bilirubin and liver enzymes started to rise and then decreased gradually to the previous normal range. The secondary workup for liver disease was unremarkable. The patient improved and was discharged. Six-month follow-up showed regression of the duodenal lesion and reduction in the common bile duct dilatation. Excluding malignancy remains the main challenge in managing groove pancreatitis, and a conservative approach is more reasonable in cases with a typical profile.


Ali et al.: Grove Pancreatitis and Ampullary Carcinoma

\section{Introduction}

Acute pancreatitis is an acute inflammation of the pancreas. The exact pathophysiology of pancreatic damage is not completely understood, but several theories have been postulated depending on the underlying cause [1]. In a typical case, the diagnosis is made based on meeting two of three criteria: acute onset of persistent, severe, epigastric pain often radiating to the back, elevation in pancreatic enzymes at least three times the upper limit of normal, and characteristic radiological features of acute pancreatitis on imaging [2]. The most common identifiable causes for acute pancreatitis are biliary and gall bladder disease accounting for around $28 \%$ of cases followed by alcohol (19\%) [3]. Patients who survive acute pancreatitis are at risk of progression to chronic pancreatitis, and alcoholics, and smokers are at greater risk [3].

First described by Becker and Mischke [4] in 1973, groove pancreatitis, also known as paraduodenal pancreatitis, is an uncommon form of chronic pancreatitis seen in $19.5 \%$ of surgical specimens of pancreatic resection. It affects the "groove," the area between the pancreatic head, duodenum, and common bile duct (CBD). It is typically seen in men in their 50 s and who are alcoholics.

Clinically, groove pancreatitis presentation includes abdominal pain and weight loss, which masquerade cancer. Moreover, it is difficult to distinguish it from other neoplastic processes involving the surrounding structures like duodenal peri-ampullary or pancreatic tumors. Final diagnosis can be challenging as it is difficult to distinguish groove pancreatitis from neoplasia even with advanced imaging like MRI and this might lead to an invasive procedure or surgery for definitive diagnosis [5].

\section{Case Report}

We are reporting a 52-year-old male with a past medical history of hypertension, asthma, smoking and known alcoholism for the past 15 years with previous episodes of acute pancreatitis less severe than this presentation. He presented to the Emergency Department because of 4 days of severe dull epigastric pain radiating to the back associated with nausea, darkening in urine color, loss of appetite, but no vomiting. The pain was related to food and was relieved with fasting. He had a non-intentional 7-kg weight loss over the last 3 months. His previous weight was $72 \mathrm{~kg}$, on admission it was $65 \mathrm{~kg}$. Physical examination was unremarkable apart from upper abdominal tenderness on palpation.

Initial lab results showed total bilirubin $43 \mu \mathrm{mol} / \mathrm{L}(0-21 \mu \mathrm{mol} / \mathrm{L})$, direct bilirubin 32 $\mu \mathrm{mol} / \mathrm{L}(\mu \mathrm{mol} / \mathrm{L}$ 0-3.4), initial liver function ALP $343 \mathrm{U} / \mathrm{L}$ (40-129 U/L), ALT $801 \mathrm{U} / \mathrm{L}$ (0-41 U/L), AST $723 \mathrm{U} / \mathrm{L}(0-40 \mathrm{U} / \mathrm{L})$, amylase 1,257 U/L (13-53 U/L), lipase >3,000 IU/L (13-60 $\mathrm{U} / \mathrm{L}$ ), triglyceride $0.8 \mathrm{mmol} / \mathrm{L}$ (normal: $<1.7 \mathrm{mmol} / \mathrm{L}$, borderline: $1.7-2.2 \mathrm{mmol} / \mathrm{L}$, high: $2.2-$ $5.6 \mathrm{mmol} / \mathrm{L}$ ), ethanol $<2.2 \mathrm{mmol} / \mathrm{L}$ (critical high $>45.0 ; 10.9-21.7 \mathrm{mmol} / \mathrm{L}$ : flushing, slowing of reflexes, impaired visual acuity; $>21.7 \mathrm{mmol} / \mathrm{L}$ : depression of CNS; $>86.8 \mathrm{mmol} / \mathrm{L}$ : fatalities reported), CA 19-9. 97.4 U/mL (0.0-27.0 U/mL), CEA $3 \mu \mathrm{g} / \mathrm{L}$ (smoker: 5.5-6.5 $\mu \mathrm{g} / \mathrm{L}$, nonsmokers: $3.8-5.0 \mu \mathrm{g} / \mathrm{L})$.

He was clinically stable with no end-organ damage and did not receive any antibiotics. Initially, he was nil per mouth and gradually started to tolerate oral feeding. During the hospital stay, the patient developed clinical jaundice and dark red urine for 4 days, which resolved gradually without any intervention. Then, bilirubin and liver enzymes followed the pattern shown in Figure 1. 
Further workup with abdominal ultrasound showed that the gallbladder was highly distended and no obvious stones could be imaged; no wall thickening and no pericholecystic fluid was noted. Sludge was noted in the lumen. Cystic duct and CBD were dilated. CBD measured $1.4 \mathrm{~cm}$. Significant intra- and extra-hepatic biliary radical dilatation was noted with associated dilatation of the pancreatic duct. Next, an abdominal computed tomography scan with contrast showed no dilated intra-hepatic biliary ducts with associated distended gallbladder. Common hepatic duct, CBD, and pancreatic duct were dilated. The pancreas was uniformly iso-dense without any obvious necrosis or hemorrhage. No obvious hyper-dense stone was seen in the biliary tract. No calcification in the pancreas or pancreatic ductal stones were seen. There is a focal iso-dense opacity mass of $23 \times 21 \mathrm{~mm}$ at the junction of the pancreatic duct with the distal end of the CBD in the pancreatic head/periampullary region with dilatation of the pancreatic duct, features suggestive of a focal small iso-dense mass in the head of the pancreas with obstruction, and the possibility of carcinoma of the head of the pancreas/periampullary mass. Next, abdominal magnetic resonance imagining (MRI) with contrast showed mural wall thickening of the second part of the duodenum with a maximum thickness of $13 \mathrm{~mm}$ at the periampullary region and narrowed lumen with the possibility of extension to the proximal 3rd and distal 1st duodenum parts. There is evidence of diffusion restriction and thickened wall enhancement. There is back pressure and significant dilatation of the CBD, intra-hepatic radicles and pancreatic ducts. The CBD measures $17.5 \mathrm{~mm}$ and the pancreatic duct $8 \mathrm{~mm}$. Gallbladder is very distended with sludge. The head of the pancreas is inseparable from duodenum wall thickening; however, there was no definite pancreatic parenchyma signal change to suggest infiltration or suspicious lesion. There is intra-hepatic radicle dilatation but no suspicious focal lesion, CBD and pancreatic duct obstruction. MR appearances are suggestive of duodenum neoplasm with ampullary involvement. There are retro-pancreatic, portal hepatic and retrocrural lymph nodes measuring up to $9 \mathrm{~mm}$ in the short axis. Then, he was scheduled for oesophagogastroduodenoscopy, which showed severe reflux esophagitis and the stomach was grossly normal, with no retained secretions. The first part of the duodenum was normal, the second part circumferential infiltrative with oedematous mucosa. The scope passed with slight negotiation to the third part. The biopsy report from the second part of the duodenum showed no evidence of parasites, increased intraepithelial lymphocytes, granulomas, metaplasia, dysplasia, or malignancy. Endoscopic ultrasound revealed a hypo-echoic circumferential lesion of the duodenal wall measuring $13 \mathrm{~mm}$ in maximal depth and limited to the submucosal layer of the duodenal wall. The interface between the lesion and the pancreas was intact. Minimal fluid was seen around the lesion. A single $11 \times 6 \mathrm{~mm}$ lymph node adjacent to the lesion was appreciated. The CBD was dilated to $11 \mathrm{~mm}$. Peri-pancreatic inflammation around the pancreatic head was shown, and the pancreatic parenchyma was normal in echotexture and pattern. The pancreatic duct was dilated to $5 \mathrm{~mm}$. The endoscopic diagnosis was as follows: duodenal polypoidal circumferential lesion limited by the submucosa (Fig. 2). The lesion is highly suspicious for a duodenal polyp with malignant degeneration. A dilated biliary system was reported (CBD and pancreatic duct). Endoscopic ultrasound-guided biopsy showed normal duodenal mucosa with no evidence of parasites, increased intraepithelial lymphocytes, granulomas, metaplasia, dysplasia, or malignancy.

Then positron emission tomography (PET) scan was done and showed CBD and pancreatic duct dilatation. Mild uptake (SUVmax 4.0) is seen with a metabolic diameter of approximately $1.5-2 \mathrm{~cm}$ in the periampullary location at the beginning of the dilated pancreatic duct.

No increased uptake was found in a 2-cm sized hypo-dense area in the pancreatic head. Physiological uptake and excretion were seen in the gastrointestinal and genitourinary tract. The liver, spleen, right adrenal glands, gallbladder, and kidneys appear grossly unremarkable. 


\section{Case Reports in Gastroenterology}

Case Rep Gastroenterol 2020;14:306-314

DOI: $10.1159 / 000507430$

c) 2020 The Author(s). Published by S. Karger AG, Basel www.karger.com/crg

Ali et al.: Grove Pancreatitis and Ampullary Carcinoma

There is no nodal hypermetabolism or obvious adenopathy in the abdomen, pelvis and inguinal region. Mild periampullary uptake is suspicious for low-grade malignancy. No PET sign of lymph node or distant involvement was found. Focal uptake in the left parotid indicated likely a Warthin tumour. Follow-up after 6 months with MR cholangiopancreatography showed regression of mural wall thickening of the second part of the duodenum at the periampullary region, associated with partial narrowing of the lumen, diffusion restriction and thickened wall enhancement. The head of the pancreas is inseparable from duodenum wall thickening. However, there was no definite pancreatic parenchyma signal change to suggest infiltration or suspicious lesion. There is a regression of CBD dilatation and intra-hepatic radicles, however, no appreciable changes with regard to the pancreatic duct. CBD measures $9 \mathrm{~mm}$ and pancreatic duct $8.7 \mathrm{~mm}$. The gallbladder is distended with normal wall thickness, no intraluminal stones or pericholecystic fluid. The liver shows normal signal intensities with significant intra-hepatic radicle dilatation, but no suspicious focal lesion. As for the abdominal lymph nodes: there are few subcentimetrr retropancreatic and portal hepatic lymph nodes; the largest measures $9 \mathrm{~mm}$ in the short axis at the portahepatis region.

\section{Discussion}

The presence of obstructive jaundice with radiological features of mass lesion in a patient with pancreatitis is a red flag for malignancy. Clinicians should include pseudo-tumour conditions like autoimmune pancreatitis, groove pancreatitis and focal chronic pancreatitis in the differential diagnosis [6].

The patient has a typical profile of groove pancreatitis: male, smoker, and alcoholic. The mass seen in the imaging was suspicious for neoplasm. Nevertheless, the natural history of malignancy is usually progressive; the decrease in the dilatation of CBD from 17.5 to $11 \mathrm{~mm}$ over 10 days and then to $9 \mathrm{~mm}$ over 6 months, and the resolution of obstructive jaundice within few days all points against cancer. Additionally, the presence of jaundice is a poor prognostic factor for ampullary carcinoma [7]. Clinically, the pain and the jaundice were improving, and the patient was able to feed orally after a few days, which is unlikely in case of duodenal cancer-causing obstruction to resolve spontaneously. Likewise, the regression of the inflammatory reaction on the follow-up supports a more benign condition.

The difficulty was there because cancer cannot be rolled out without a histopathologic diagnosis. So, the patient underwent biopsy on oesophagogastroduodenoscopy and fine-needle aspiration with endoscopic ultrasound both did not show any evidence of neoplasia or atypical cells. The finding of duodenal polypoidal circumferential lesion limited by the submucosa seen in the MRI (Fig. 2) and the endoscopic ultrasound most probably represents Brunner's gland hyperplasia which is typical of groove pancreatitis [8] supported by the positron image result showing minimal regional uptake (Fig. 3).

The MRI finding of duodenal wall thickening and luminal stenosis are going with groove pancreatitis [9]. The finding of retroperitoneal lymph nodes in MRI and the history of weight loss make the suspicion of malignancy more likely. However, the PET scan showed that these lymph nodes had no uptake and the duodenal lesion has a mild uptake, which cannot rule out low-grade ampullary carcinoma [10], but make it less likely as PET is more sensitive than CT and MRI in diagnosing locoregional ampullary cancer [11].

The occurrence of jaundice in the setting of pancreatitis without obvious biliary disease is usually either due to liver injury or associated obstruction in the CBD due to oedema of the surrounding structures [12]. In this patient, the associated increase in liver enzymes with 
Ali et al.: Grove Pancreatitis and Ampullary Carcinoma

mixed picture make hepatocellular injury more likely. Secondary workup for the raised liver enzymes and bilirubin was done and revealed, anti-mitochondrial $\mathrm{Ab}$ negative, anti-smooth muscle $A b$ negative, ANA CTD Int negative, hepatitis $B$ surface antigen negative hepatitis $C A b$, negative ceruloplasmin $31 \mathrm{mg} / \mathrm{dL}$ (15-30 mg/dL) alpha-1-antitrypsin $183.5 \mathrm{mg} / \mathrm{dL}$ (90.0$200.0 \mathrm{mg} / \mathrm{dL}$ ).

The raised liver enzymes and bilirubin followed the same time frame (Fig. 1) and got back to the previous normal level before the current illness. This indicates that the injury is secondary to the underlying illness (pancreatitis itself) as the workup was negative, and the patient did not receive antibiotics or other drugs that may precipitate liver injury.

We conclude that groove pancreatitis is an uncommon form of pancreatitis and should be considered; it is probably unrecognized due to the wrong attribution of alcohol as the cause of the condition, which is common among this group of patients. The great challenge in the condition is rolling out malignancy, which necessitates a more aggressive approach for reaching a diagnosis. However, in a typical case with a characteristic radiological finding, it might be wiser to follow a more conservative approach, waiting for regression of the inflammatory process, which is highly supportive for the diagnosis and reassuring, so avoiding surgery and its complications.

\section{Acknowledgment}

We wish to show our gratitude to the Internal Medicine Residency Program, to Dr. Ahmed Ali Almohammed, Dr. Dabia Hamad Almohanadi and Qatar National Library for their scientific support.

\section{Statement of Ethics}

This case was approved by the Hamad Medical Corporation Research Center, and consent was obtained from the patient.

\section{Disclosure Statement}

All authors have no conflict of interest.

\section{Funding Sources}

The study was supported by the Qatar National Library.

\section{Author Contributions}

Elrazi Ali: writing and editing, final approval.

Akhnuwkh Jones: clinical care, final approval.

Ahmed Mahfouz: writing the radiology part, editing, final approval. 
Mohamed Yassin: writing and editing, final approval.

Abdelatif Abdelmola: writing and editing, final approval.

\section{References}

1 Wang GJ, Gao CF, Wei D, Wang C, Ding SQ. Acute pancreatitis: etiology and common pathogenesis. World J Gastroenterol. 2009 Mar;15(12):1427-30.

2 Banks PA, Bollen TL, Dervenis C, Gooszen HG, Johnson CD, Sarr MG, et al.; Acute Pancreatitis Classification Working Group. Classification of acute pancreatitis-2012: revision of the Atlanta classification and definitions by international consensus. Gut. 2013 Jan;62(1):102-11.

3 Yadav D, O'Connell M, Papachristou GI. Natural history following the first attack of acute pancreatitis. Am J Gastroenterol. 2012 Jul;107(7):1096-103.

4 Becker V, Mischke U. Groove pancreatitis. Int J Pancreatol. 1991 Nov-Dec;10(3-4):173-82.

5 Blasbalg R, Baroni RH, Costa DN, Machado MC. MRI Features of Groove Pancreatitis. AJR Am J Roentgenol. 2007 Jul;189(1):73-80.

6 Madhani K, Farrell JJ. Autoimmune Pancreatitis: An Update on Diagnosis and Management. Gastroenterol Clin North Am. 2016 Mar;45(1):29-43.

7 Kamisawa T, Tu Y, Egawa N, Nakajima H, Horiguchi S, Tsuruta K, et al. Clinicopathologic features of ampullary carcinoma without jaundice. J Clin Gastroenterol. 2006 Feb;40(2):162-6.

8 Adsay NV, Zamboni G. Paraduodenal pancreatitis: a clinico-pathologically distinct entity unifying "cystic dystrophy of heterotopic pancreas", "para-duodenal wall cyst", and "groove pancreatitis". Semin Diagn Pathol. 2004 Nov;21(4):247-54.

9 Blasbalg R, Baroni RH, Costa DN, Machado MC. MRI features of groove pancreatitis. AJR Am J Roentgenol. 2007 Jul;189(1):73-80.

10 Delbeke D, Martin WH. Update of PET and PET/CT for hepatobiliary and pancreatic malignancies. HPB (Oxford). 2005;7(3):166-79.

11 Na SJ, Kim SH, Choi WH, Lee SY, Chung SK. Usefulness of 18F-FDG PET/CT in the diagnosis of locoregional periampullary cancer. J Nucl Med. 2009;50(suppl 2):1761.

12 McCollum WB, Jordan PH Jr. Obstructive jaundice in patients with pancreatitis without associated biliary tract disease. Ann Surg. 1975 Aug;182(2):116-20. 


\section{Bilirubin T, Alk Phos, ALT, \& AST}

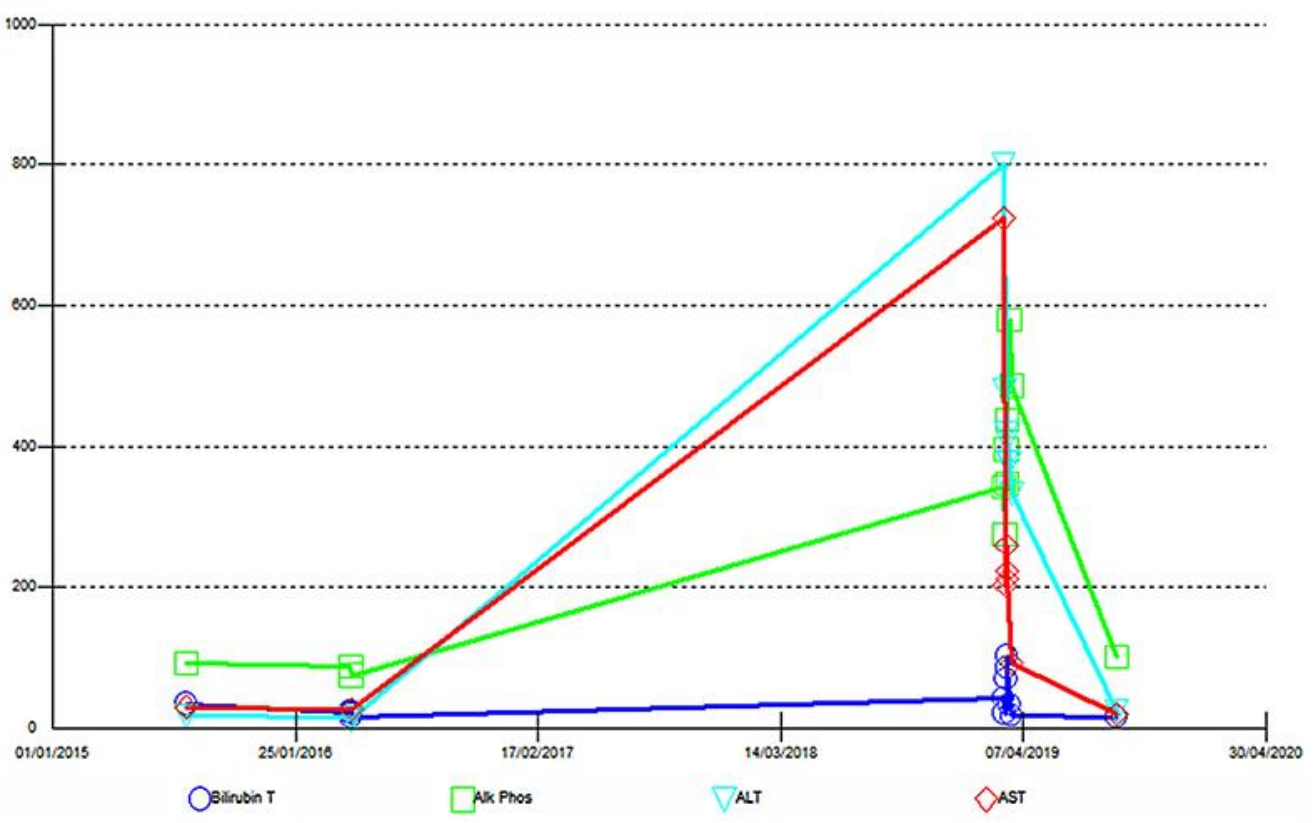

Fig. 1. The transient rise in bilirubin and liver enzymes during the illness. 


\section{Case Reports in Gastroenterology}

Ali et al.: Grove Pancreatitis and Ampullary Carcinoma
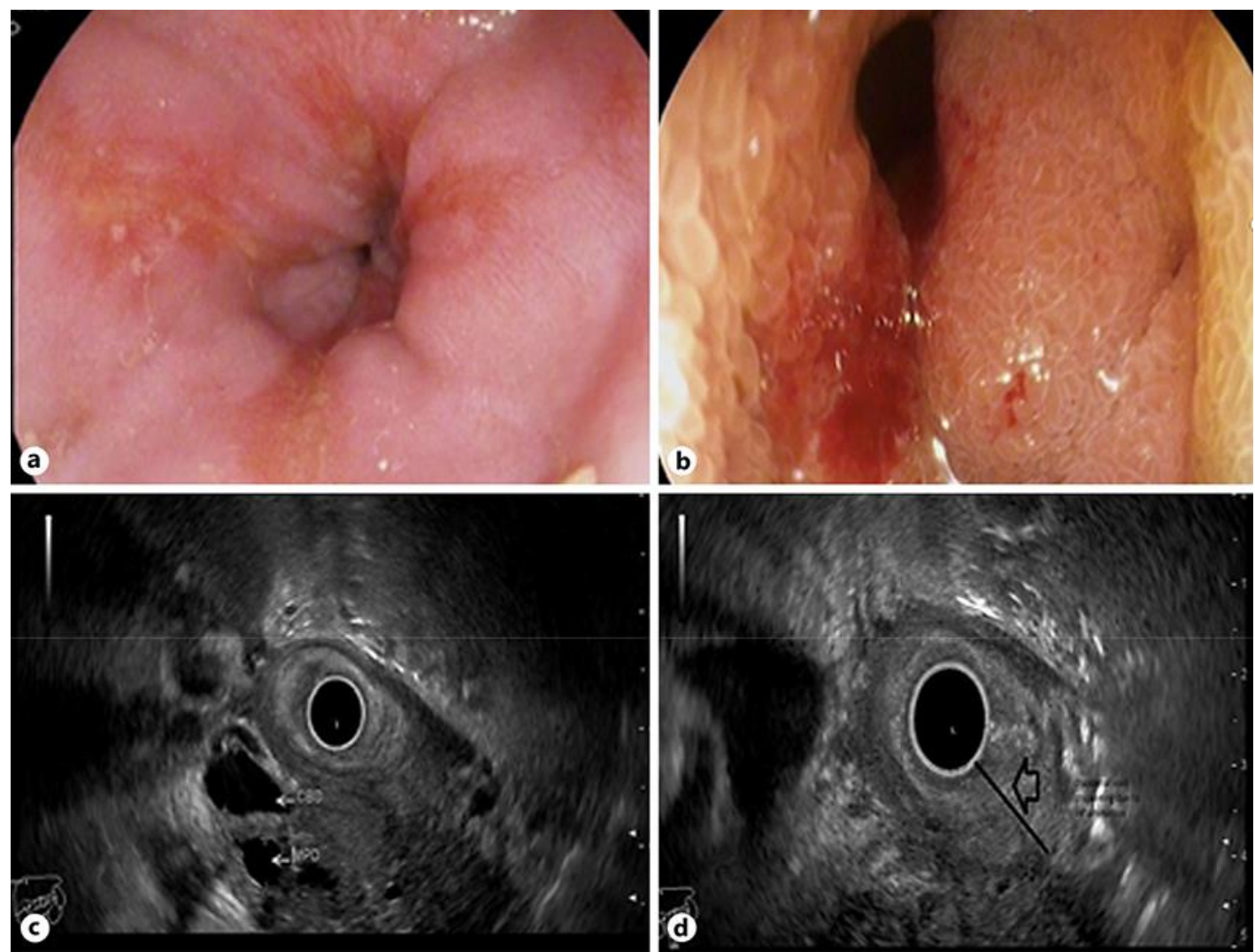

Fig. 2. a, b The esophagogastroduodenoscopy of the second part of the duodenum showing luminal narrowing. $\mathbf{c}, \mathbf{d}$ The endoscopic ultrasound of the second part of the duodenum showing dilated common bile duct and main pancreatic duct with duodenal wall thickening. 

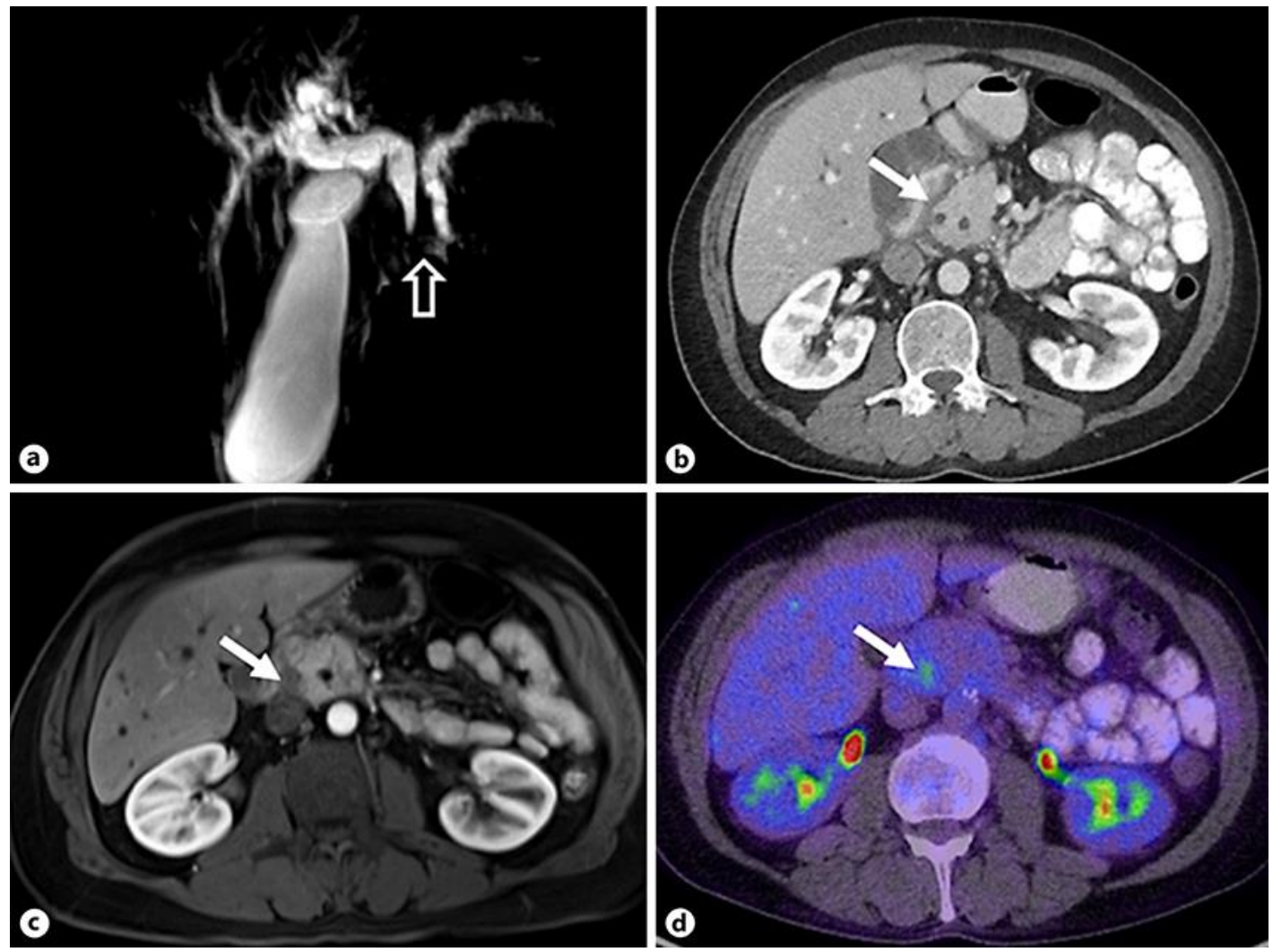

Fig. 3. MRCP (a) shows dilatation of the pancreatic duct, the bile ducts and the gall bladder due to obstruction at the level of the pancreatic head (hollow arrow), forming the double-duct sign, which is suggestive of malignant pancreatic head neoplasm. Contrast-enhanced CT (b) gadolinium-enhanced MRI (c) and PET/CT (d) showed no evidence of neoplasm at the pancreatic head and showed, instead, inflammatory reaction at the groove between the pancreatic head and duodenum evident by a poorly enhancing tissue on CT and MRI and corresponding poor uptake of 18F-FDG on PET/CT (solid arrows). 